\title{
THE STATIONARY LOCAL SOJOURN TIME IN SINGLE SERVER TANDEM QUEUES WITH RENEWAL INPUT
}

\author{
PIERRE LE GALL \\ France Telecom, CNET \\ 4 Parc de la Bérengère \\ F-92210 Saint-Cloud, France
}

(Received November, 1998; Revised June, 1999)

\begin{abstract}
We start from an earlier paper evaluating the overall sojourn time to derive the local sojourn time in stationary regime, in a single server tandem queue of $(m+1)$ stages with renewal input. The successive service times of a customer may or may not be mutually dependent, and are governed by a general distribution which may be different at each sage.
\end{abstract}

Key words: Tandem Queue, First Come-First Served Discipline, Renewal Input.

AMS subject classifications: $60 \mathrm{~K} 25,90 \mathrm{~B} 22$.

\section{Introduction}

We consider the stochastic behavior of a single server tandem queue with $(m+1)$ successive queues and with a renewal customer arrival process. For a customer, the successive service times may or may not be different and may or may not be mutually dependent. They are governed, at each stage, by a general distribution which may be different. Customers enter the queue of a given stage immediately on terminating their service at the previous stage. No limitation is placed on the length of the waiting time with buffers being supposed of infinite capacity. Service discipline at each stage is "first come-first served" (FC-FS). We wish to derive the distribution function of the local sojourn time in stationary regime.

For practical applications, we will consider the case of Poisson arrivals at the first stage. More particularly, we will present the calculations in the case of several packet traffic streams, with each traffic stream packet length (i.e., service duration) being constant (i.e., deterministic).

For this paper, we will refer to our earlier publications (see Le Gall [1-3]). 


\section{Notation and Assumptions}

The number of successive queues is $(m+1)$. At stage $\boldsymbol{k}(k=1,2, \ldots, m+1)$, for the $n$th customer, we define the following times:

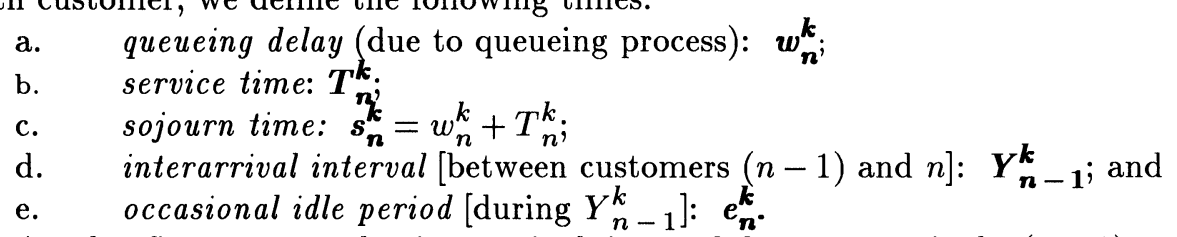

At the first queue, the interarrival interval between arrivals $(n-1)$ and $n$ is: $\boldsymbol{Y}_{\boldsymbol{n}-\mathbf{1}}^{\mathbf{1}} \equiv \boldsymbol{Y}_{\boldsymbol{n}-\mathbf{1}}$. The arrival process is a renewal process with successive intervals $\boldsymbol{Y}_{n-1}$ being independent and identically distributed with distribution function $\boldsymbol{F}_{0}(t)$. Service times $\boldsymbol{T}_{\boldsymbol{n}}^{\boldsymbol{k}}$ (for $\boldsymbol{n}$ given) are assumed to be independent of the arrival process with distribution function $\boldsymbol{F}_{\boldsymbol{k}}(\boldsymbol{t})$ at stage $\boldsymbol{k}$, which is the same for all customers. We introduce the Laplace-Stieltjes (L-S) transforms, for $R e(z) \geq 0$ :

$$
\begin{aligned}
& E \exp \left(-z Y_{n-1}\right)=\varphi_{0}(z)=\int_{0}^{\infty} e^{-z t} \cdot d F_{0}(t) \\
& E \exp \left(-z T_{n}^{k}\right)=\varphi_{k}(z)=\int_{0}^{\infty} e^{-z t} \cdot d F_{k}(t)
\end{aligned}
$$

We may note the relations (at stage $\boldsymbol{k}$ ):

$$
\begin{gathered}
Y_{n-1}^{k}=T_{n}^{k-1}+e_{n}^{k-1} \\
w_{n}^{k}=\operatorname{Max}\left[0, s_{n-1}^{k}-Y_{n-1}^{k}\right]=\operatorname{Max}\left[0, s_{n-1}^{k}-\left(T_{n}^{k-1}+e_{n}^{k-1}\right)\right] .
\end{gathered}
$$

We let:

$$
\begin{gathered}
T_{n}^{1}+\ldots+T_{n}^{m}=\boldsymbol{T}_{n}^{\prime}(\boldsymbol{m}) \\
s_{n}^{2}+\ldots+s_{n}^{m+1}=\boldsymbol{S}_{n}^{m}
\end{gathered}
$$

Finally, in stationary regime, we let:
a. arrival rate: $\lambda=\left[E Y_{n-1}\right]^{-1}$;
b. load (i.e., traffic intensity): $\rho_{k}=\lambda \cdot E\left[T_{n}^{k}\right]$.

\section{Preliminary Results in Tandem Queues}

\subsection{The General Recurrence Relation}

In Le Gall [1], Theorem (A.1), we presented the following properties:

Theorem 1: (Recurrence relation) If at stage $\boldsymbol{k}(k=2 \ldots m+1)$ we have the relation: 


$$
T_{n}^{k-1} \leq s_{n-1}^{k}
$$

the following recurrence relation using (3) is then satisfied for arbitrary arrival processes (excluding simultaneous arrivals):

$$
\left(T_{n}^{1}+w_{n}^{2}\right)+\ldots+\left(T_{n}^{m}+w_{n}^{m+1}\right)=\operatorname{Max}\left[T_{n}^{\prime}(m), S_{n-1}^{m}-e_{n}^{1}\right]
$$

Property 1: (Busy Period not Broken Up) Equation (5) means that the busy period is not broken up at stage $k$, during the busy period at stage $(k-1)$. It follows that, for the occasional idle periods $e_{n}^{k}$, we have: $e_{n}^{1}=\ldots=e_{n}^{m+1}$. Finally, only $\boldsymbol{e}_{\boldsymbol{n}}^{\mathbf{1}}$ appears in (6).

Property 2: (Includes Intermediate Arrivals) An intermediate arrival at stage $\boldsymbol{k}$ may be considered as an arrival at stage 1 with $T_{n}^{1}=\ldots=T_{n}^{k-1}=0$. Equation (5) being satisfied, (6) includes the case of intermediate arrivals.

Property 3: (Includes Successive Service Times Mutually Independent when Heavy Traffic) If the load (i.e., traffic intensity) is high enough to increase $w_{n-1}^{k}$ such that (5) is almost always satisfied during busy periods, the above theorem can be applied to the case of mutually independent successive service times.

Finally, (6) may be of general use.

\subsection{The Equivalent Packet Tandem Queue}

In Le Gall [1] and [2], we have seen that the local sojourn time distribution is practically defined by the two first moments, and finally by $\operatorname{Var} T_{n}^{\prime}(m)$. Consequently, in Le Gall [2] we introduced the parameter $\boldsymbol{m}_{\mathbf{0}}$, with

$$
\operatorname{Var}\left(m_{0} \cdot T_{n}^{m+1}\right)=\operatorname{Var} T_{n}^{\prime}(m),
$$

and excluding the case of $T_{n}^{m+1}$ and $T_{n}^{\prime}(m)$ constant. In this way, the local sojourn distribution is practically the same as for a single server packet tandem queue corresponding to identical, successive service times: $T_{n}^{1}=\ldots=T_{n}^{m_{0}}=T_{n}^{m_{0}+1^{1}}$, if $\boldsymbol{m}_{\mathbf{0}}$ is an integer. From (7), (6) becomes, with (3):

$$
S_{n}^{m_{0}}=\operatorname{Max}\left[m_{0} \cdot T_{n}^{m_{0}+1}, S_{n-1}^{m_{0}}-e_{n}^{1}\right] .
$$

Finally, due to (5), the local sojourn time distribution is practically given by the equivalent packet tandem queue above for $\left(\boldsymbol{m}_{\mathbf{0}}+\mathbf{1}\right)$ stages. When $\boldsymbol{m}_{\mathbf{0}}$ is not an integer, the distribution function may be used with this new value $\boldsymbol{m}_{\mathbf{0}}$.

When service times are highly varying, we have: $\left[E T_{n}^{m}+{ }^{1}\right]^{2}<<E\left[T_{n}^{m+1}\right]^{2}$ and $\left[E T_{n}^{\prime}(m)\right]^{2}<<E\left[T_{n}^{\prime}(m)\right]^{2}$. It follows: $\operatorname{Var} T_{n}^{m+1} \cong E\left[T_{n}^{m+1}\right]^{2}$ and $\operatorname{Var} T_{n}^{\prime}(m) \cong$ $E\left[T_{n}^{\prime}(m)\right]^{2}$. Consequently, (7) yields

$$
m_{0}^{2}=\frac{E\left[T_{n}^{\prime}(m)\right]^{2}}{E\left[T_{n}^{m+1}\right]^{2}}
$$

\subsection{The Agglutination Phenomenon}

For the equivalent packet tandem queue, (2) gives: 


$$
s_{n}^{m+1}=\operatorname{Max}_{[}\left[T_{n}^{m+1}, s_{n-1}^{m+1}-e_{n}^{m}\right] .
$$

During a busy period, at stages $(m+1)$ and $m$, we have $e_{n}^{m}=0$, and consequently $s_{n}^{m+1}=s_{n-1}^{m+1}=\ldots=s_{n_{1}}^{m+1}$, where $n_{1}$ corresponds to the arbitrary customer initiating the busy period at these successive stages. Thus, the local sojourn time appears to be constant during any busy period, and equal to the sojourn time of the customer initiating the busy period. In the case of the arrival of a new customer with a service duration higher than $s_{n_{1}}^{m+1}$, the busy period is broken up, and this new customer initiates a new busy period. From stage to stage, busy periods initiated by long service durations tend to amalgamate, with busy periods initiated by short service durations, leading to some increase of the sojourn time. Finally, from stage to stage, the agglutination phenomenon is amplified, with the local sojourn time of short service durations being equal to long service durations corresponding to customers with no local queueing delay. As a consequence, with (5) of busy periods not broken $u p$, the product form theory cannot exist.

We may note that, in Sections 3.1-3.3, the arrival process (at stage 1) is not necessarily restricted to a renewal process. This process may be arbitrary in stationary regime.

\subsection{The Overall Distribution in Case of a Renewal Input}

Now, we come back to the case of a renewal input, and we consider only the equivalent packet tandem queue (i.e., $T_{n}^{m+1}=T_{n}^{1}$ ). We let, in stationary regime, for $\operatorname{Re}(z) \geq 0$ :

$$
\begin{gathered}
\operatorname{Prob}\left(w_{n}^{1}=0\right)=Q_{1}, \\
E \exp \left(-z s_{n}^{1}\right)=\Phi_{1}(z), \\
\varphi_{1}(z ; t)=\int_{0}^{t} e^{-z \alpha} \cdot d F_{1}(\alpha), \\
\operatorname{Prob}\left(S_{n}^{m}<t\right)=S_{m}(t),
\end{gathered}
$$

where $S_{n}^{m}$ is defined by (3). To present the following expressions, we will use Cauchy contour integrals along the imaginary axis in the complex plane $\boldsymbol{u}$. If the contour (followed from the bottom to the top) is to the right of the imaginary axis (the contour being closed at infinity to the right), we write $\int_{0}$. If the contour is to the left of the imaginary axis, we write $\int_{-0}$. We introduce: to

$$
\boldsymbol{Q}_{\mathbf{1}}(\boldsymbol{t})=\operatorname{Exp}\left\{\frac{-1}{2 \pi i} \int_{-0} \log \left[1-\varphi_{0}(-u) \cdot \varphi_{1}(u, t)\right] \cdot \frac{d u}{u}\right\}
$$

In Le Gall [3], and with (5) of busy periods not broken up, we gave the following expression for the distribution function of the overall sojourn time, from stages 2 to $(m+1)$ : 
with

$$
S_{\boldsymbol{m}}(t)=\frac{1}{2 \pi i} \cdot \int_{+0} \frac{\varphi_{0}(-u) \cdot \Phi_{1}(u)}{Q_{1}} \cdot S_{m}(t, u) \cdot \frac{d u}{u}
$$

$$
\begin{gathered}
S_{m}(t, u)=v_{0}\left(\frac{t}{m}\right) \cdot\left[v\left(\frac{t}{m}, u\right)\right]^{m}, \quad v_{0}(t)=\frac{Q_{1}}{Q_{1}(t)} \cdot F_{1}(t) \\
v(t, u)=\operatorname{Exp}\left\{-u \int_{t}^{\infty} \frac{1-F_{1}(v)}{Q_{1}(v)} \cdot d v\right\}
\end{gathered}
$$

by using (1), (10) and (11).

\subsection{The Unitary Sojourn Time}

In the stationary regime, it may be useful to introduce the concept of unitary sojourn time $\boldsymbol{U}(\boldsymbol{m})$ (i.e., the overall sojourn time divided by $\boldsymbol{m}$ [number of equivalent stages]). From (12) and (13), and assuming (5) of busy periods not broken up, the distribution function of $U(m)$ is:

with

$$
\boldsymbol{U}_{\boldsymbol{m}}(\boldsymbol{t})=\frac{1}{2 \pi i} \int_{+0} \frac{\varphi_{0}(-u) \cdot \Phi_{1}(u)}{Q_{1}} \cdot u_{m}(t, u) \cdot \frac{d u}{u}
$$

$$
\boldsymbol{u}_{\boldsymbol{m}}(\boldsymbol{t}, \boldsymbol{u})=v_{0}(t) \cdot[v(t, u)]^{m}
$$

or

$$
\boldsymbol{u}_{\boldsymbol{m}}(\boldsymbol{t}, \boldsymbol{u})=u_{1}(t, u) \cdot[v(t, u)]^{m-1}
$$

where $\boldsymbol{v}_{\mathbf{0}}(\boldsymbol{t})$ and $\boldsymbol{u}_{1}(\boldsymbol{t}, \boldsymbol{u})$, defined by (13), relates only to the case $m=1$. Here, $\boldsymbol{u}_{\boldsymbol{m}}(\boldsymbol{t}, \boldsymbol{u})$ is the distribution function of the random variable $\boldsymbol{U}(\boldsymbol{m}, \boldsymbol{u})$. In other words, we have the stochastic expression:

$$
\boldsymbol{U}(\boldsymbol{m})=\frac{1}{2 \pi i} \cdot \int_{+0} \frac{\varphi_{0}(-u) \cdot \Phi_{1}(u)}{Q_{1}} \cdot U(m, u) \cdot \frac{d u}{u} .
$$

We note that $\boldsymbol{v}_{\mathbf{0}}(\boldsymbol{t})$ and $\boldsymbol{v}(\boldsymbol{t}, \boldsymbol{u})$ are the distribution functions of $\boldsymbol{V}_{\mathbf{0}}$ and $\boldsymbol{V}_{\boldsymbol{j}}(\boldsymbol{u})$, $j=1, \ldots, m$, respectively. From (15), we write:

$$
\boldsymbol{U}(\boldsymbol{m}, \boldsymbol{u})=\operatorname{Min}_{j=1 \ldots m}\left[V_{0}, V_{j}(u)\right] .
$$

Finally, from (18) we deduce the stochastic expression giving the overall sojourn time:

$$
S_{n}^{m}=m \cdot U(m)=\frac{1}{2 \pi i} \cdot \int_{+0} \frac{\varphi_{0}(-u) \cdot \Phi_{1}(u)}{Q_{1}} \cdot[m \cdot U(m, u)] \cdot \frac{d u}{u},
$$

which has $\boldsymbol{U}_{\boldsymbol{m}}\left(\frac{\boldsymbol{t}}{\boldsymbol{m}}\right)$, from (14), for its distribution function.

We now consider the case of Poisson input. Since the arrival rate, for an arbitrary 
customer, is $\lambda$, we have:

$$
\varphi_{0}(z)=\frac{\lambda}{\lambda+z}, \quad \rho=\lambda \cdot E\left(T_{n}\right) \quad(\rho<1) .
$$

In the integrand of (19) there is just one pole for $\operatorname{Re}(u)>0: u=\lambda$. Equations (14) and (15) become, for the distribution function of the unitary sojourn time:

$$
\boldsymbol{U}_{\boldsymbol{m}}(\boldsymbol{t})=u_{m}(t, \lambda)=v_{0}(t) \cdot[v(t, \lambda)]^{m}, \text { with } Q_{1}=1-\rho .
$$

\section{The Local Sojourn Time in Case of a Renewal Input}

In stationary regime with renewal input, the local sojourn time $\boldsymbol{s}_{\boldsymbol{n}}^{\mathbf{m}+\mathbf{1}}$, at stage $(m+1)$, may be deduced from stochastic equation (19), assuming (5) of busy periods not broken up to be able to use the equivalent packet tandem queue [where $\boldsymbol{m}$ is the value of $\boldsymbol{m}_{\mathbf{0}}$ of $\left.(9)\right]$ :

$$
s_{n}^{m+1}=S_{n}^{m}-S_{n}^{m-1}=\frac{1}{2 \pi i} \cdot \int_{+0} \frac{\varphi_{0}(-u) \cdot \Phi_{1}(u)}{Q_{1}} \cdot D(m, u) \cdot \frac{d u}{u},
$$

with $D(m, u)=m \cdot U(m, u)-(m-1) \cdot U(m-1, u)$.

From (18) we write:

$$
\begin{gathered}
D(m, u)=\operatorname{Min}\left[m \cdot V_{m}(u), m \cdot U(m-1, u)\right]-(m-1) \cdot U(m-1, u) \\
=\operatorname{Min}\left[m \cdot V_{m}(u)-(m-1) \cdot U(m-1, u), U(m-1, u)\right] .
\end{gathered}
$$

The distribution function $\boldsymbol{d}_{\boldsymbol{m}}(\boldsymbol{t}, \boldsymbol{u})$ of $D(m, u)$ corresponds to the inequalities;

$$
U(m-1, u)<t, m \cdot V_{m}(u)-(m-1) \cdot U(m-1, u)<t .
$$

These inequalities are satisfied by the expression:

$$
\boldsymbol{d}_{\boldsymbol{m}}(\boldsymbol{t}, \boldsymbol{u})=\int_{0}^{t} v\left(\frac{t+w}{m}, u\right) \cdot d_{w} u_{m-1}\left(\frac{w}{m-1}, u\right)
$$

where $\boldsymbol{v}(\boldsymbol{t}, \boldsymbol{u})$ and $\boldsymbol{u}_{\boldsymbol{m}-\mathbf{1}}(\boldsymbol{t}, \boldsymbol{u})$ are defined by (13) and (16), respectively. Finally, from $(22)$ and (24) we can state:

Theorem 2: (Local sojourn time) In stationary regime, with (5) of busy periods not broken up, and a number $(m+1)$ of equivalent stages [as defined by the value of $m_{0}$ in (9)], the distribution function of the local sojourn time $[$ at stage $(m+1)]$ for an arbitrary customer is:

$$
\boldsymbol{s}(\boldsymbol{t}, \boldsymbol{m}+\mathbf{1})=\frac{1}{2 \pi i} \cdot \int_{+0} \frac{\varphi_{0}(-u) \cdot \Phi_{1}(u)}{Q_{1}} d_{m}(t, u) \cdot \frac{d u}{u}
$$


where $\boldsymbol{d}_{\boldsymbol{m}}(\boldsymbol{t}, \boldsymbol{u})$ is given by $(24)$.

It may be very useful to simplify $(24)$ of $\boldsymbol{d}_{\boldsymbol{m}}(\boldsymbol{t}, \boldsymbol{u})$. When $\boldsymbol{m}$ increases, we have for $\boldsymbol{t}$ given

$$
v\left(\frac{t+w}{m}, u\right) \rightarrow v(0, u)
$$

Equation (24) becomes:

$$
\boldsymbol{d}_{\boldsymbol{m}}(\boldsymbol{t}, \boldsymbol{u}) \rightarrow v(0, u) \cdot u_{m-1}\left(\frac{t}{m-1}, u\right)
$$

We can state:

Corollary 1: (Approximation) When $\boldsymbol{m}$ increases, expression $\boldsymbol{d}_{\boldsymbol{m}}(\boldsymbol{t}, \boldsymbol{u})$ in $(25)$ becomes:

$$
\boldsymbol{d}_{\boldsymbol{m}}(\boldsymbol{t}, \boldsymbol{u}) \cong v(0, u) \cdot u_{m-1}\left(\frac{t}{m-1}, u\right)
$$

where $\boldsymbol{v}(\mathbf{0}, \boldsymbol{u})$ and $\boldsymbol{u}_{\boldsymbol{m}-\mathbf{1}}(\boldsymbol{t}, \boldsymbol{u})$ are defined by (13) and (15), respectively. Thus

$$
u_{m-1}\left(\frac{t}{m-1}, u\right)=v_{0}(t) \cdot\left[v\left(\frac{t}{m-1}, u\right)\right]^{m-1},
$$

with $\boldsymbol{v}_{\mathbf{0}}(\boldsymbol{t})$, as defined by (13), being related only to stage 1.

Note: In the numerical example provided below in Section 5.3, we will see that the approximation is already excellent for $m=2$.

\section{The Local Sojourn Time in Case of a Poisson Input}

\subsection{The Distribution Function}

In the case of Poisson input, as already noted for (21), we have one pole $u=\lambda$ (for $R e(u)>0)$ in the integrand of $(25)$. We deduce

$$
\boldsymbol{s}(\boldsymbol{t}, \boldsymbol{m}+\mathbf{1})=d_{m}(t, \lambda)
$$

and for (26)

$$
\boldsymbol{s}(\boldsymbol{t}, \boldsymbol{m}+1) \cong v_{0}(t) \cdot\left[v\left(\frac{t}{m-1}, \lambda\right)\right]^{m-1} \cdot v(0, \lambda)
$$

\subsection{Case of Packet Traffics with Poisson Input}

As an important example, consider (in stationary regime) the case of a total traffic stream with $\boldsymbol{N}$-component, partial Poisson traffic streams labeled $j(j=1 \ldots N)$. For traffic stream $\boldsymbol{j}$, packet lengths are constant (i.e., deterministic), and equal to $\boldsymbol{T}_{\boldsymbol{j}}$ $\left(T_{1}<T_{2}<\ldots<T_{N}\right)$. The partial arrival rate is $\boldsymbol{\lambda}_{\boldsymbol{j}}$, and the total arrival rate (for an arbitrary customer) is: $\lambda=\sum_{j=1}^{N} \lambda_{j}$. The partial loads are $\boldsymbol{\rho}_{\boldsymbol{j}}=\lambda_{j} \cdot T_{j}$, with a total load: $\boldsymbol{\rho}=\sum_{j=1}^{N} \rho_{j}$. With $(1)$, we achieve, for the distribution of an arbitrary 
customer (i.e., packet) and for $\operatorname{Re}(z) \geq 0$ :

$$
\varphi_{1}(z)=\sum_{j=1}^{N} \frac{\lambda_{j}}{\lambda} \cdot e^{-z T} j
$$

In Le Gall [1], formula (B.3), we gave the expression:

$$
Q_{1}(t)=1-\lambda \cdot \int_{0}^{t} u \cdot d F_{1}(u), \quad Q_{1}(\infty)=Q_{1}\left(T_{N}\right)=1-\rho .
$$

The distribution function of the service times are as follows:

For $t<T_{1}$ :

$$
F_{1}(t)=0, \quad Q_{1}(t)=1
$$

For $T_{k}<t<T_{k+1}$ :

$$
F_{1}(t)=\frac{\lambda_{1}+\ldots+\lambda_{k}}{\lambda}, \quad Q_{1}(t)=1-\left(\rho_{1}+\ldots+\rho_{k}\right) .
$$

\section{a. Exact expression for the mean local sojourn time}

From (21), the distribution function of the unitary sojourn time is:

$$
\boldsymbol{u}_{\boldsymbol{m}}(\boldsymbol{t}, \lambda)=v_{0}(t) \cdot[v(t, \lambda)]^{m},
$$

where $v_{0}(t)$ and $v(t, \lambda)$ are defined by (13). We deduce for (32):

For $t<T_{1}$ :

$$
u_{m}(t, \lambda)=0
$$

For $T_{k}<t<T_{k+1}$ :

$$
u_{m}(t, \lambda)=v_{0}(t) \cdot[v(t, \lambda)]^{m}
$$

with

$$
v_{0}(t)=\frac{\lambda_{1}+\ldots+\lambda_{k}}{\lambda} \cdot \frac{1-\rho}{1-\left(\rho_{1}+\ldots+\rho_{k}\right)}
$$

and

$$
\begin{aligned}
& v(t, \lambda)= \\
& \operatorname{Exp}\left\{-\left[\frac{\lambda_{k+1}+\ldots+\lambda_{N}}{1-\left(\rho_{1}+\ldots+\rho_{k}\right)} \cdot\left(T_{k+1}-t\right)+\ldots+\frac{\lambda_{N}}{1-\left(\rho_{1}+\ldots+\rho_{N-1}\right)} \cdot\left(T_{N}-T_{N-1}\right)\right]\right\} .
\end{aligned}
$$

We deduce the mean overall sojourn time, from stage 2 to stage $(m+1)$ : 


$$
E\left(S_{n}^{m}\right)=m \cdot \int_{0}^{T_{N}}\left[1-u_{m}(t, \lambda)\right] \cdot d t
$$

and we deduce the mean local sojourn time at stage $(m+1)$, for an arbitrary packet:

$$
s(m+1)=m \cdot \int_{0}^{T_{N}}\left[1-u_{m}(t, \lambda)\right] \cdot d t-(m-1) \cdot \int_{0}^{T_{N}}\left[1-u_{m-1}(t, \lambda)\right] \cdot d t .
$$

\section{b. Approximated expression for the local sojourn time distribution}

Approximated equation (29) gives for the distribution function of the local sojourn time [at stage $(m+1)$ ], for an arbitrary packet:

For $t<T_{1}$ :

$$
s(t, m+1)=0
$$

For $T_{k}<t<T_{k+1}$ :

$$
s(t, m+1)=v(0, \lambda) \cdot v_{0}(t) \cdot\left[v\left(\frac{t}{m-1}, \lambda\right)\right]^{m-1}=v_{0}(t) \cdot\left[v\left(\frac{t}{m}, \lambda\right)\right]^{m},
$$

where $\boldsymbol{v}_{\mathbf{0}}(\boldsymbol{t})$ and $\boldsymbol{v}(\boldsymbol{t}, \boldsymbol{\lambda})$ are given by $(33)$; and

For $t>T_{N}$ :

$$
s(t, m+1)=1
$$

From (36), we get an approximated value for the mean local sojourn time at stage $(m+1)$ :

$$
\boldsymbol{s}_{\mathbf{1}}(\boldsymbol{m}+\mathbf{1})=\int_{0}^{T_{N}}[1-s(t, m+1)] \cdot d t
$$

In the same way, we get the second moment:

$$
\left.m_{2} m+1\right)=2 \cdot \int_{0}^{T_{N}} t \cdot[1-s(t, m+1)] \cdot d t
$$

the variance

$$
v_{1}(m+1)=m_{2}(m+1)-\left[s_{1}(m+1)\right]^{2},
$$

and the standard deviation

$$
\sigma_{1}(m+1)=\sqrt{v_{1}(m+1)} .
$$

To evaluate the accuracy of (36), we compare (35) for $\boldsymbol{s}(\boldsymbol{m}+\mathbf{1})$, and (37) for $\boldsymbol{s}_{\mathbf{1}}(\boldsymbol{m}+\mathbf{1})$ with numerical values. 


\subsection{An Example of Three Packet Traffic Streams}

For numerical calculations, we will consider the case of three Poisson packet traffic streams. Here $\boldsymbol{v}_{\mathbf{0}}(\boldsymbol{t})$ and $\boldsymbol{v}(\boldsymbol{t}, \boldsymbol{\lambda})$, used in (36), become:

For $T_{1}<t<T_{2}$ :

$$
\begin{gathered}
v_{0}(t)=\frac{\lambda_{1}}{\lambda} \cdot \frac{1-\rho}{1-\rho_{1}} \\
\boldsymbol{v}(\boldsymbol{t}, \lambda)=\operatorname{Exp}\left\{-\left[\frac{\lambda_{2}+\lambda_{3}}{1-\rho_{1}} \cdot\left(T_{2}-t\right)+\frac{\lambda_{3}}{1-\left(\rho_{1}+\rho_{2}\right)} \cdot\left(T_{3}-T_{2}\right)\right]\right\}
\end{gathered}
$$

For $T_{2}<t<T_{3}$ :

$$
\begin{gathered}
v_{0}(t)=\frac{\lambda_{1}+\lambda_{2}}{\lambda} \cdot \frac{1-\rho}{1-\left(\rho_{1}+\rho_{2}\right)} \\
\boldsymbol{v}(\boldsymbol{t}, \boldsymbol{\lambda})=\operatorname{Exp}\left\{-\frac{\lambda_{3}}{1-\left(\rho_{1}+\rho_{2}\right)} \cdot\left(T_{3}-t\right)\right\} .
\end{gathered}
$$

Table 1 gives $\boldsymbol{s}(\boldsymbol{m}+\mathbf{1}), \boldsymbol{s}_{\mathbf{1}}(\boldsymbol{m}+\mathbf{1})$ and $\boldsymbol{\sigma}_{\mathbf{1}}(\boldsymbol{m}+\mathbf{1})$, related to (35), (37), and (39) respectively, for $m=1,2,3,5,10$ and 15 . We observe that $\boldsymbol{s}_{\mathbf{1}}(\boldsymbol{m}+\mathbf{1})$ is approximately equal to the exact value $\boldsymbol{s}(\boldsymbol{m}+1)$ for $m>1$, and equal for $m=1$. When $\boldsymbol{m}$ increases, $\sigma_{1}(m+1) \rightarrow 0$, and consequently $s(m+1) \rightarrow T_{3}$, as explained by the agglutination phenomenon (see Section 3.3).

\begin{tabular}{|c|c|c|c|}
\cline { 2 - 4 } \multicolumn{1}{c|}{} & exact & \multicolumn{2}{c|}{ approximated } \\
\hline$m$ & $s(m+1)$ & $s_{1}(m+1)$ & $\sigma_{1}(m+1)$ \\
\hline 1 & 14.7 & 14.7 & 12.8 \\
\hline 2 & 18.9 & 19.2 & 12.6 \\
\hline 3 & 21.8 & 22.4 & 11.6 \\
\hline 5 & 25.5 & 26.1 & 9.0 \\
\hline 10 & 28.9 & 29.3 & 4.1 \\
\hline 15 & 29.7 & 29.9 & 1.8 \\
\hline
\end{tabular}

Table 1: Local Queue Distribution [at stage $(m+1)$ ] for an Arbitrary Packet 1st approximation: formulae (40).

Example: $(3$ packet traffic streams $): \quad N=3 ; T_{1}=1, T_{2}=5, \quad \boldsymbol{T}_{\mathbf{3}}=\mathbf{3 0} ; \rho_{1}=$ $\rho_{2}=\rho_{3}=0.2(\rho=0.6)$.

Mean local queue:

Exact value: $\boldsymbol{s}(\boldsymbol{m}+1)$, Equation (35);

Approximated value: $\boldsymbol{s}_{\mathbf{1}}(\boldsymbol{m}+\mathbf{1})$, Equation (37).

Standard deviation:

Approximated value: $\boldsymbol{\sigma}_{\mathbf{1}}(\boldsymbol{m}+\mathbf{1})$, Equation (39). 


\section{A General Approximation in Case of Poisson Input}

In (36) we may write, for the long service durations:

For $T_{n-1}<t<T_{N}$ :

$$
\boldsymbol{s}_{\mathbf{2}}(\boldsymbol{t}, \boldsymbol{m}+\mathbf{1})=\left(1-\frac{\lambda_{N}}{\lambda}\right) \cdot \frac{1-\rho}{1-\left(\rho-\rho_{N}\right)} \cdot \operatorname{Exp}\left\{-\frac{m \rho_{N}}{1-\left(\rho-\rho_{N}\right)} \cdot\left(1-\frac{t}{m T_{N}}\right)\right\}
$$

For $t>T_{N}$ :

$$
s_{2}(t, m+1)=1
$$

where the set $\left(\lambda_{N}, \rho_{N}, T_{N}\right)$ relates to the longest packets.

In Table 2, we use this approximated distribution function for $0<t<T_{N}$, independent of $T_{1}$ and $T_{2}$. We observe the accuracy of this approximation by comparing the exact value $s(m+1)$, and the new approximated value $s_{2}(m+1)$ deduced from (37) by using (41):

$$
\boldsymbol{s}_{\mathbf{2}}(\boldsymbol{m}+\mathbf{1})=\int_{0}^{T_{N}}\left[1-s_{2}(t, m+1)\right] \cdot d t
$$

\begin{tabular}{|c|c|c|c|}
\cline { 2 - 4 } \multicolumn{1}{c|}{} & exact & \multicolumn{2}{c|}{ approximated } \\
\hline$m$ & $s(m+1)$ & $s_{2}(m+1)$ & $\sigma_{2}(m+1)$ \\
\hline 3 & 21.8 & 21.5 & 13.0 \\
\hline 5 & 25.5 & 25.6 & 10.2 \\
\hline 10 & 28.9 & 29.2 & 4.8 \\
\hline 15 & 29.7 & 29.8 & 2.1 \\
\hline
\end{tabular}

Table 2: Local Queue Distribution [at stage $(m+1)$ ] for an Arbitrary Packet 2nd approximation: (41), using Example of Table 1.

Mean local queue: $\quad$ Exact value: $\boldsymbol{s}(\boldsymbol{m}+1)$, Equation (35);

Approximated value: $\boldsymbol{s}_{\mathbf{2}}(\boldsymbol{m}+\mathbf{1})$, Equation (42).

Standard deviation: Approximated value: $\sigma_{2}(\boldsymbol{m}+1)$.

In the same way, an analogous expression to (39) gives the standard deviation $\sigma_{2}(\boldsymbol{m}+1)$. In Table 2, the accuracy of $\boldsymbol{s}_{2}(\boldsymbol{m}+1)$ is good for $m>2$, but the relative error of $\sigma_{2}(m+1)$ stands between $10 \%$ and $15 \%$. For a first use, it is sufficient. The agglutination phenomenon, presented in Section 3.3, explains that the value $T_{N}$ alone appears: we have seen that busy periods initiated by $T_{N}$ tend to amalgamate busy periods initiated by $T_{j}(j<N)$. We state for any distribution function of the service durations, in case of a finite support:

Property 4: (Impact of longest service times) In stationary regime and in case of Poisson input with (5) of busy periods not broken up, and a number $(\boldsymbol{m}+1)$ of equivalent stages as defined by (9), the distribution function of the local sojourn time 
[at stage $(m+1)$ ] for an arbitrary customer can be approximated (for $0<t<T_{N}$ ) by (41), when the support of this distribution is finite.

\section{Conclusion}

Finally, Equation (5) of busy periods not broken up leads us to a general simplified (41) to easily evaluate the distribution of the local sojourn time in tandem queues, in the case of a finite support for the distribution of the successive service times of a customer (services which are different and which may or may not be mutually dependent).

\section{References}

[1] Le Gall, P., Traffic modeling in packet switched networks for single links, Annales des Telecom 49:3-4 (1994), 111-126.

[2] Le Gall, P., Bursty traffic in packet switched networks, Proc. ITC-14 (Antibes, France, June 1994), The Fund. Role of Teletraffic in the Evolution of Telecom. Networks, Elsevier Science B.V., 1a (1994), 535-549.

[3] Le Gall, P., The overall sojourn time in tandem queues with identical successive service times and renewal input, Stoch. Proc. and their Appl. 52 (1994), 165178. 


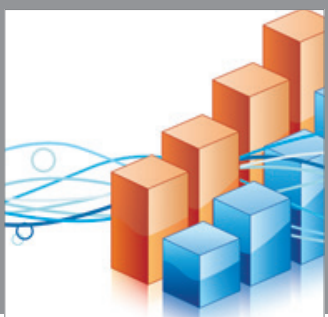

Advances in

Operations Research

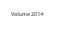

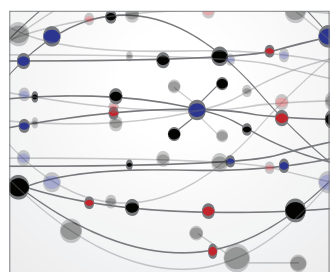

\section{The Scientific} World Journal
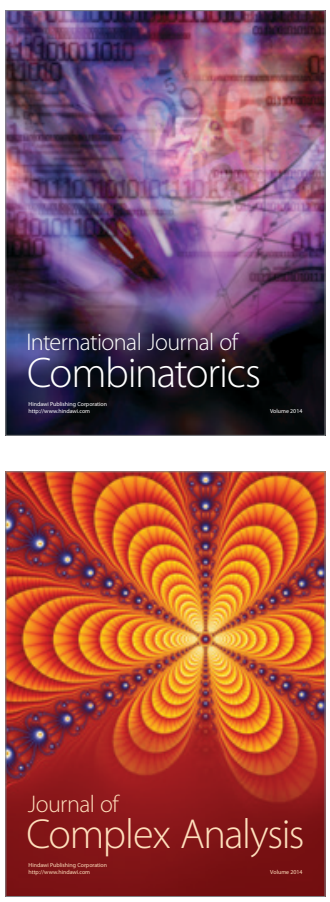

International Journal of

Mathematics and

Mathematical

Sciences
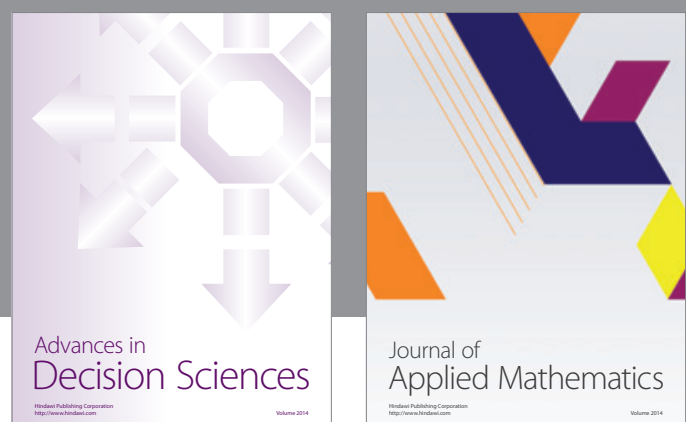

Journal of

Applied Mathematics
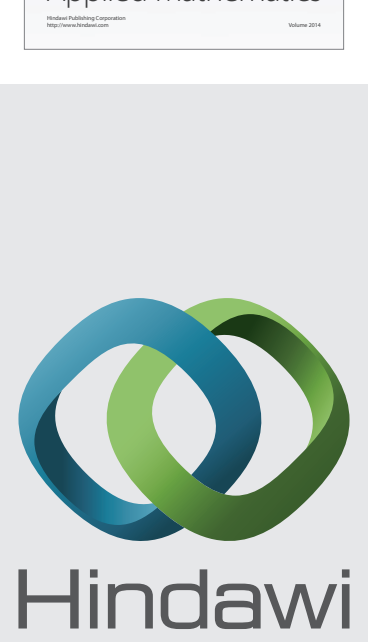

Submit your manuscripts at http://www.hindawi.com
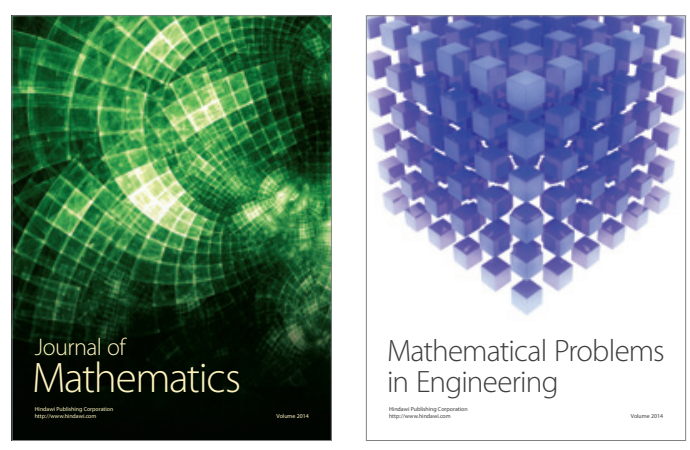

Mathematical Problems in Engineering
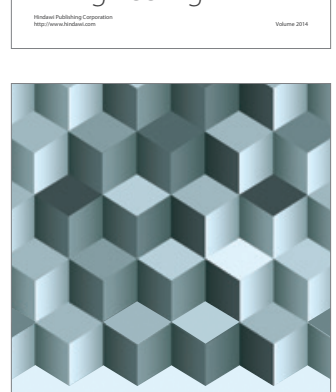

Journal of

Function Spaces
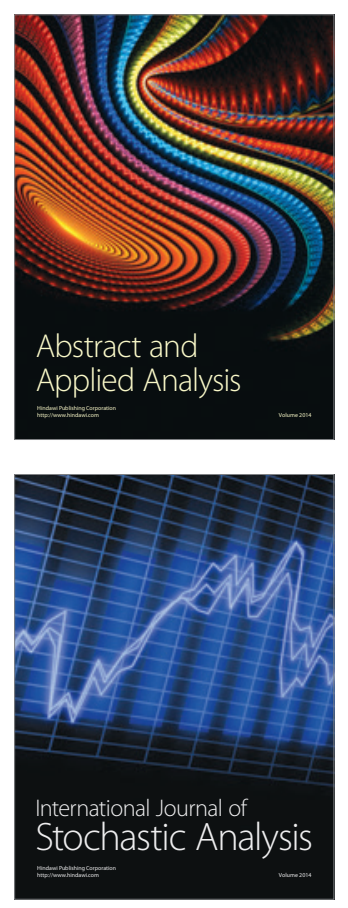

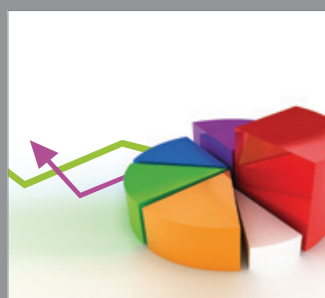

ournal of

Probability and Statistics

Promensencen
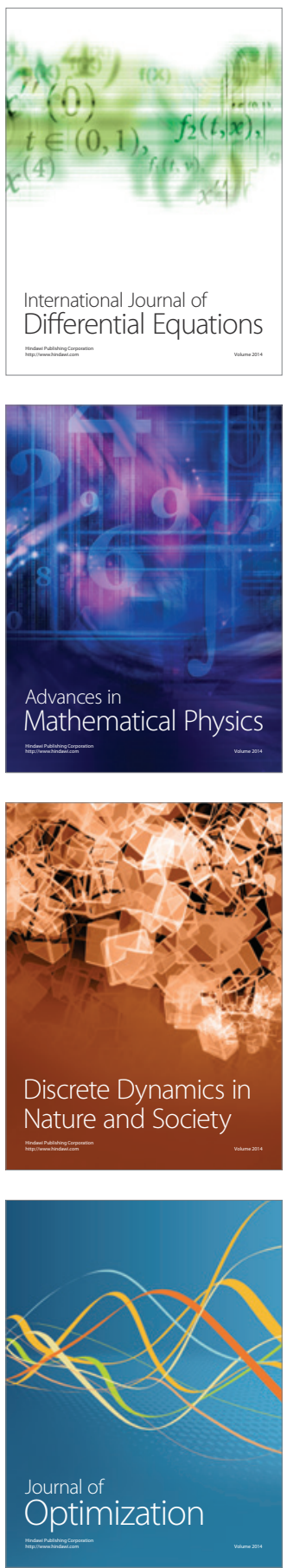